\title{
Can the contralateral scapula be used as a reliable template to reconstruct the eroded scapula during shoulder arthroplasty?
}

\author{
Filip Verhaegen, $M D^{a, 1, *}$, Katrien Plessers, Ir, MSc ${ }^{b, c, 1}$, Olivier Verborgt, MD, PhD ${ }^{d}$, \\ Lennart Scheys, Ir, PhDa ${ }^{a}$ Philippe Debeer, MD, PhDa
}

\author{
${ }^{a}$ Department of Development and Regeneration, KU Leuven, Division of Orthopaedics, Institute for Orthopaedic Research \\ and Training (IORT), Pellenberg, Belgium \\ ${ }^{b}$ Department of Biomechanics, KU Leuven, Leuven, Belgium \\ ${ }^{c}$ Materialise NV, Leuven, Belgium \\ ${ }^{d}$ Department of Orthopedic Surgery, AZ Monica Hospital and University Hospital of Antwerp, Deurne, Belgium
}

\begin{abstract}
Hypothesis: The contralateral scapula can be used as a reliable template to determine scapular offset, glenoid inclination, and version of the native scapula in view of reconstructing pathologic scapulae.

Methods: Three-dimensional measurements of scapular offset, inclination, and version were performed using data from a set of 50 bilateral computed tomography scans of full scapulae to determine direct sideto-side differences.

Results: The scapula pairs had a mean bilateral difference of $2 \mathrm{~mm}$ in offset, $2^{\circ}$ in inclination, and $2^{\circ}$ in version. Ninety percent of the scapula pairs showed an offset difference smaller than $3 \mathrm{~mm}$. In $96 \%$ and $94 \%$ of the scapula pairs, the inclination difference and version difference, respectively, were smaller than $5^{\circ}$. The maximum bilateral difference for offset, inclination, and version was $6 \mathrm{~mm}, 6^{\circ}$, and $8^{\circ}$, respectively. Discussion and Conclusion: The anatomic parameters of scapular offset, glenoid inclination, and version are quite symmetrical and fall into the currently technically feasible accuracy of shoulder arthroplasty implantation. The healthy scapula can be used as a template to guide the reconstruction of the glenoid during shoulder arthroplasty planning in the case of unilateral advanced arthropathy.

Level of evidence: Anatomy Study; Imaging

(C) 2018 Journal of Shoulder and Elbow Surgery Board of Trustees. All rights reserved.

Keywords: Scapular morphology; glenoid erosion; version; inclination; offset; between-side difference; symmetry
\end{abstract}

\footnotetext{
Ethical committee approval was obtained from Ethische Commissie Onderzoek UZ/KU Leuven (B322201524039; study No. S57735).

${ }^{1}$ These authors have contributed equally to this work.

*Reprint requests: Verhaegen Filip, MD, Department of Development and Regeneration, KU Leuven, Division of Orthopaedics, Institute for Orthopaedic Research and Training, Weligerveld 1, B-3212 Pellenberg, Belgium.

E-mail address: filip.verhaegen@uzleuven.be (F. Verhaegen).
}

Glenohumeral arthropathy can be characterized by scapular bone loss leading to a distorted scapular morphology. ${ }^{25,32}$ With the help of modern planning and guiding techniques, surgeons can reconstruct scapular morphologic parameters such as version and inclination with an accuracy of a few degrees during shoulder arthroplasty. ${ }^{8,26}$ Optimal reconstruction of the native joint anatomy in terms of glenoid version and inclination in the case of total shoulder arthroplasty (TSA) has 
been reported to affect glenoid component survival and functional outcome. ${ }^{9,13,15,19,24,33}$ However, as scapular morphology parameters have a wide range of normal values, ${ }^{5}$ the native scapular anatomy should be known when planning and performing a TSA, which can be challenging, given the presence of substantial scapular bone loss. To overcome this, it has been suggested to use the contralateral scapula as a more "patient-specific" template., ${ }^{2,18}$

In this light, previous research has tried to investigate the intraindividual correlation of anatomic parameters of glenoid morphology such as version and inclination. Most of this research only reported means and no direct side-to-side differences, therefore limiting its potential for further clinical use. Furthermore, the reported values were determined with the help of standard radiographic or single-slice, 2-dimensional (2D) computed tomography (CT) scan measurements. ${ }^{2,6,18,21,31,34}$ These techniques have poor reliability as a result of variations in the gantry angle at the time of CT or plain-film acquisition. ${ }^{3,7,17,22,23,27,29}$ In contrast, 2D measurements in which the 2D images are first corrected to the plane of the scapula by 3-dimensional (3D) reconstruction have shown lower variability. ${ }^{4,716,20}$ Nevertheless, with this method, we still have to choose a certain 2D slice for measurements. This inevitably results in intraobserver and interobserver variability and high labor intensity, further limiting its clinical application potential. ${ }^{1,4,10}$ More automated 3D measurements can overcome these problems. ${ }^{12}$

The goal of this study was to determine, with the help of direct 3D measurements, whether the contralateral scapula can be used as a reliable template to determine scapular offset, glenoid inclination, and version of the native scapula in view of reconstructing pathologic scapulae. Our hypothesis was that the anatomic parameters of glenoid inclination and version and scapular offset would be symmetrical.

\section{Materials and methods}

\section{Data}

This is a prospective diagnostic study. We obtained 66 bilateral CT scans of the entire scapula from patients without glenohumeral arthropathy presenting to our outpatient clinic. The CT scan (BrightSpeed; GE Healthcare, Diegem, Belgium) parameters were as follows: $512 \times 512$ matrix, 50 -cm field of view, and slice spacing of $1.25 \mathrm{~mm}$. The CT scan images were reviewed by the first author for any signs of bone lesions (osteophytes, joint space narrowing, subchondral sclerosis, or cysts). If any bony lesion was present, the CT scan data were excluded. We finally included data from 50 bilateral CT scans of full scapulae.

\section{Measurements}

Inclination, version, and scapular offset were computed for all scapulae. To do so, the anonymized Digital Imaging and Communications in Medicine images were segmented using medical imaging

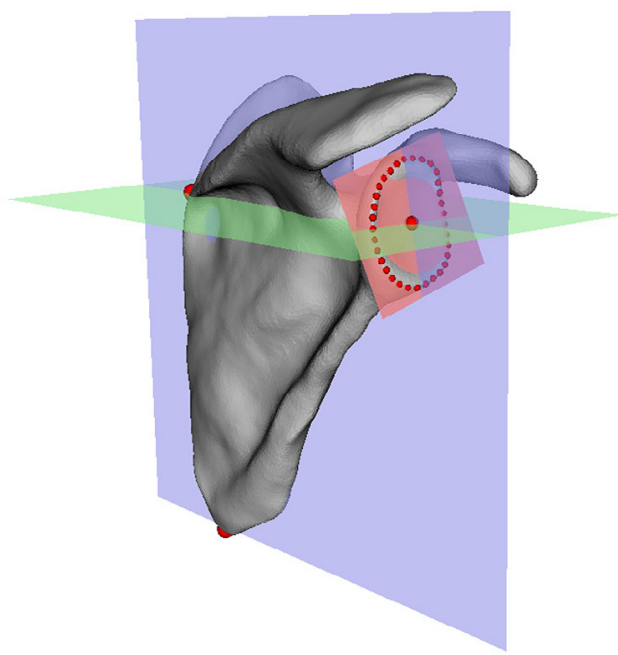

Figure 1 Three-dimensional view indicating the different planes and anatomic landmarks. The scapular plane (blue) is the plane through the trigonum spinae (upper left red dot), angulus inferior (lower red dot), and glenoid center point (upper right red dot). The axial plane (green) is the plane parallel to the trigonum spinaeglenoid center point axis and perpendicular to the scapular plane. The glenoid center point is the mean of uniformly distributed points along the rim, whereas the glenoid plane (red) is defined as the bestfitting plane through these rim points. (For interpretation of the references to color in this figure legend, the reader is referred to the web version of this article.)

software (Mimics; Materialise, Leuven, Belgium) and converted to $3 \mathrm{D}$ scapula models. Next, the 3D scapula models were uploaded in a custom-made software application, developed in Python 3.5 (Python Software Foundation, Wilmington, DE, USA), for easily indicating the required landmarks. First, the trigonum spinae and angulus inferior were indicated in accordance with International Society of Biomechanics recommendations. ${ }^{35}$ Next, the glenoid rim was identified by indicating a minimum of 10 points along the rim. To compute the glenoid plane and center point, a basis spline-a smooth curve-was fitted through the indicated rim points and automatically resampled to equally distributed points with a uniform distance of $\pm 3 \mathrm{~mm}$ between them (Fig. 1). The glenoid center point was defined as the mean point of the resampled rim points, whereas the glenoid plane was defined as the least-square best-fit plane. Furthermore, the scapular plane was formed by the glenoid center point, trigonum spinae, and angulus inferior. ${ }^{20}$ The plane through the glenoid center point and trigonum spinae and going perpendicular to the scapular plane represented the axial plane. The axis between the glenoid center point and trigonum spinae point is called the "centerline." To assess inclination and version, the glenoid plane normal was projected to the scapular plane and axial plane. Then, the angle between the projected glenoid plane normal and the centerline was measured in both planes to obtain glenoid inclination and version, respectively (Figs. 2 and 3). Last, scapular offset was computed as the distance between the glenoid center point and trigonum spinae. To assess repeatability and reliability, all landmark indications and corresponding measurements were repeated twice by 2 individual surgeons ( 1 orthopedic fellow and 1 senior resident). This resulted in 4 sets of inclination, version, and offset values for each scapula. 


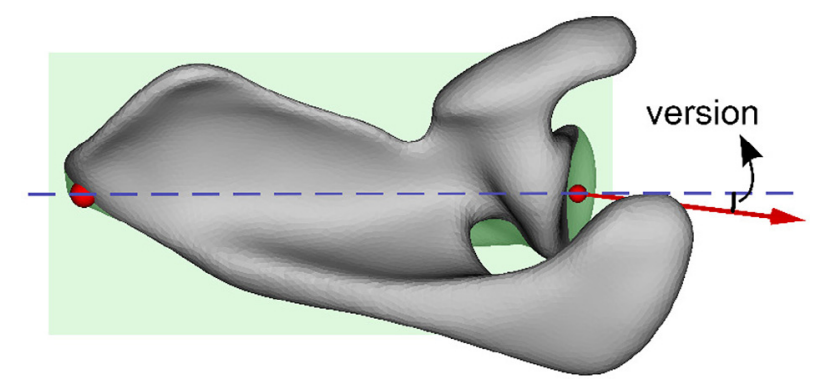

Figure 2 Version is computed as the angle between the centerline (blue line) and the glenoid plane normal projected to the axial plane (red line). (For interpretation of the references to color in this figure legend, the reader is referred to the web version of this article.)

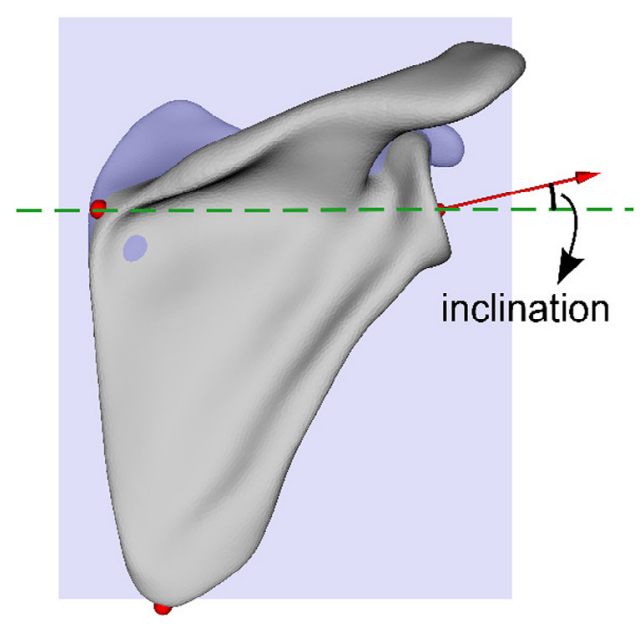

Figure 3 Inclination is computed as the angle between the centerline axis (green line) and the glenoid plane normal projected to the scapular plane (red line). (For interpretation of the references to color in this figure legend, the reader is referred to the web version of this article.)

\section{Bilateral comparison}

Inclination, version, and offset were compared within each scapula pair using the mean of the 4 operator measurements. To assess bilateral variation, these mean measurements were compared between the left and right scapulae, and the differences were reported for each scapula pair. We determined how many scapula pairs had a sideto-side difference smaller than $5^{\circ}$ for both inclination and version. We used this cutoff value based on the previously reported accuracy of implantation of glenoid components with patient-specific guides. $^{8,11,14}$

\section{Surgeon variability}

To determine the precision and variability of our method, interrater variability and intrarater variability were computed for each scapula. Inter-rater variability was expressed as the difference in the overall mean measurement of a scapula compared with the mean measurement of 1 operator. The mean measurement of an operator was defined as the mean of both of his observations. In addition, intrarater variability was expressed as the mean difference between
1 single operator's measurement and the mean measurement of that operator. Furthermore, the intraclass correlation coefficient was computed as a measure of between-subject variation as a proportion of total variation. ${ }^{30}$

\section{Results}

The resulting mean offset, inclination, and version values of all 100 scapulae were $105 \pm 8 \mathrm{~mm},-11^{\circ} \pm 4^{\circ}$, and $-7^{\circ} \pm 4^{\circ}$, respectively. The global offset, inclination, and version values were within a range of 88 to $125 \mathrm{~mm},-21^{\circ}$ to $-1^{\circ}$, and $-17^{\circ}$ to $3^{\circ}$, respectively (Fig. 4 ). The scapula pairs had a mean bilateral difference of $2 \mathrm{~mm}$ in offset, $2^{\circ}$ in inclination, and $2^{\circ}$ in version. Ninety percent of the scapula pairs showed an offset difference smaller than $3 \mathrm{~mm}$ (Fig. 5). In $96 \%$ and $94 \%$ of the scapula pairs, the inclination difference and version difference, respectively, were smaller than $5^{\circ}$. The maximum bilateral difference for offset, inclination, and version was $6 \mathrm{~mm}, 6^{\circ}$, and $8^{\circ}$, respectively. The 4 sets of measurements resulted in a mean interoperator variability error of $0.25 \mathrm{~mm}$, $0.23^{\circ}$, and $0.32^{\circ}$ and a mean intraoperator variability of $0.27 \mathrm{~mm}, 0.26^{\circ}$, and $0.23^{\circ}$ for offset, inclination, and version, respectively. The intraclass correlation coefficients were 0.995 , 0.986 , and 0.980 for offset, inclination, and version measurements, respectively (Fig. 6).

\section{Discussion}

Our results showed that the contralateral side can predict the glenoid version and inclination adequately for $94 \%$ and $96 \%$ of patients, respectively. Furthermore, the maximum betweenside difference is smaller than the reported maximum error of current implantation techniques. ${ }^{8,11,14}$ Therefore, we believe that we can confirm our main hypothesis that the anatomic parameters of glenoid inclination and version are quite symmetrical and fall into the currently technically feasible accuracy of shoulder arthroplasty implantation. Iannotti and colleagues ${ }^{10}$ developed a method to predict the native glenoid anatomy from the pathologic scapula, but they were only able to predict native glenoid version in a $5^{\circ}$ range for $58 \%$ of their patients. Other methods such as linear regression analysis of the Resch angle and anterior glenoid wall angle showed even less reliable results. ${ }^{10}$ Preoperative CT scanning of the scapula for planning of a TSA is increasingly used and will probably become standard practice for a glenoid with advanced erosion. Data for the contralateral side are readily available after CT scanning without increasing $\mathrm{x}$-ray radiation exposure. Our technique is easy applicable during 3D planning and is highly reliable in terms of interobserver and intraobserver reliability. We believe that the healthy scapula can be used as a template to guide the reconstruction of glenoid version and inclination during shoulder arthroplasty planning in the case of unilateral advanced arthropathy.

Our findings suggest that scapular offset is indeed also a quite symmetrical parameter, with a between-side difference 


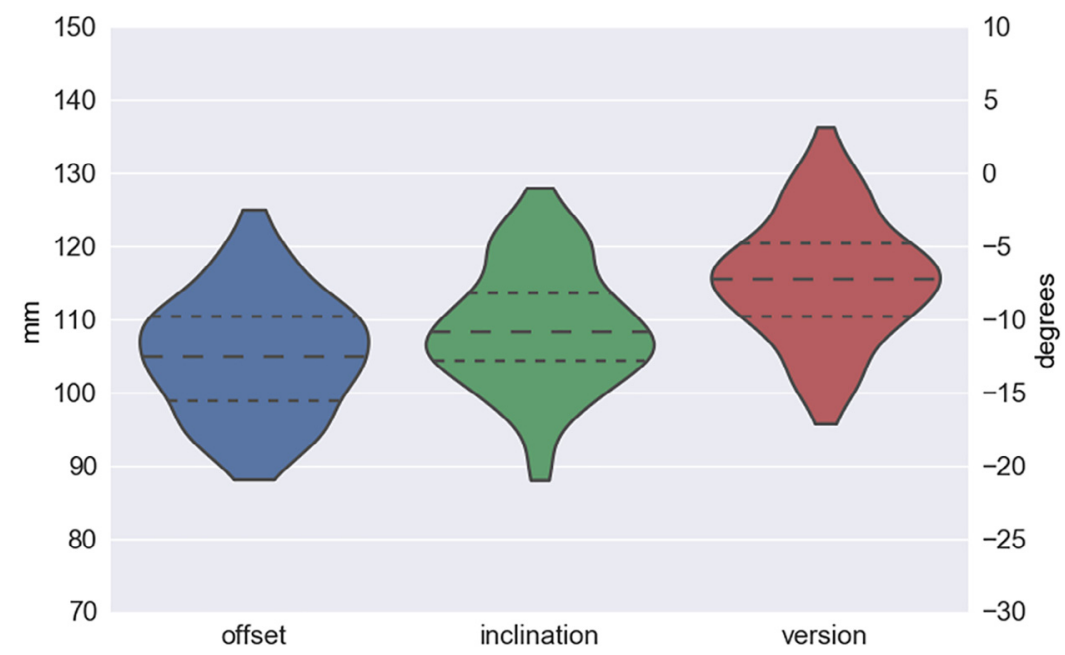

Figure 4 Violin plots showing the offset, inclination, and version distribution of the 100 scapulae. The $0.25,0.5$, and 0.75 quartile values are shown by dashed lines.

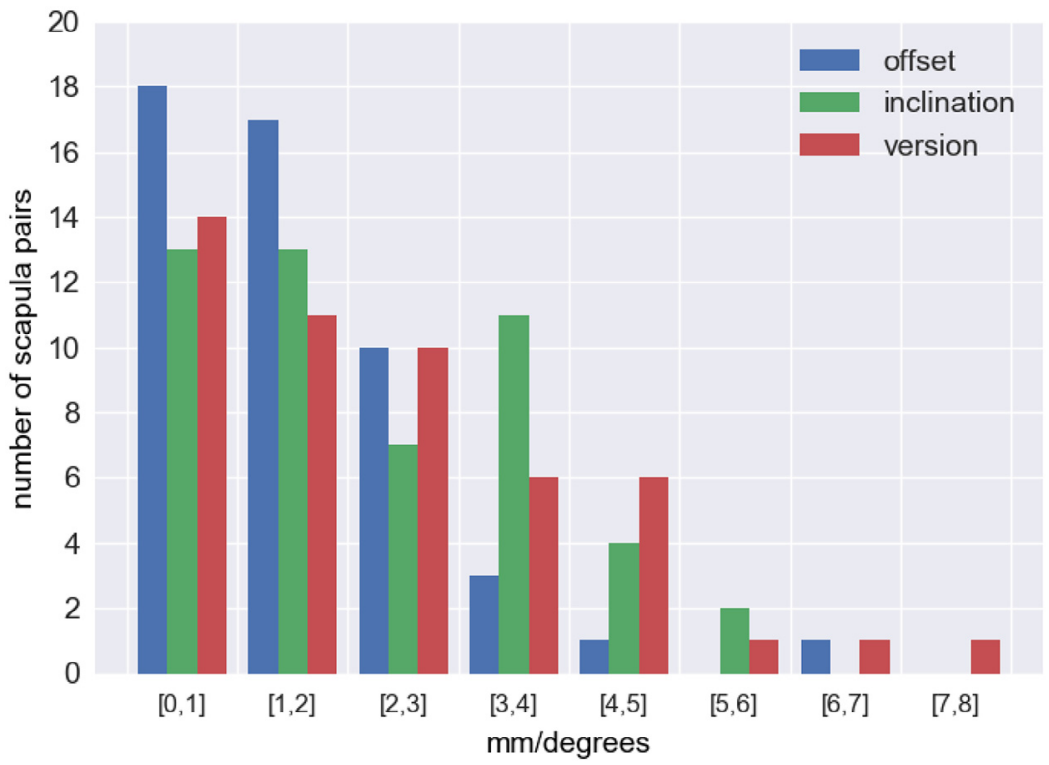

Figure 5 Histogram showing the bilateral difference between the left and right scapulae for the 50 scapula pairs.

of $3 \mathrm{~mm}$ or less in $90 \%$ of patients and $5 \mathrm{~mm}$ or less in $98 \%$ of patients. As there is no currently available literature about the limits of glenoid component medialization, further research should be undertaken to determine objective guidelines regarding the amount of medialization that can be tolerated for shoulder arthroplasty survival and functional outcome.

Our study has some limitations. We used a methodology that relies largely on manual identification of bony landmarks on 3D segmentations, which can affect the results. Segmenting bones from CT scan images has been shown to be a highly reliable technique with a root mean square error of $0.55 \mathrm{~mm} .{ }^{28}$ In our study, there was no statistical difference between the interoperator and intraoperator error for inclination and offset measurements. This finding indicates that measurements from different operators are equally as good as several measurements from a single operator. Only for version was there a clear difference between interoperator and intraoperator variability. The maximum version interoperator error was only $1.23^{\circ}$, which was extremely small and below the clinically relevant threshold. We believe this is due to both operators using a different standard when indicating the landmarks off the glenoid rim. Despite the fact that our method shows good interobserver and intraobserver reliability, further research is necessary to validate our methodology to define the glenoid plane and center point and to develop more automated software tools to further decrease the existing variability. All CT scans were obtained from patients with soft tissue problems of the shoulder and were verified for the presence of any bone lesions by an experienced shoulder surgeon (F.V.). Despite this, we cannot exclude that these soft tissue problems might have been associated with small unnoticed changes in the osseous morphology of the included scapulae. 


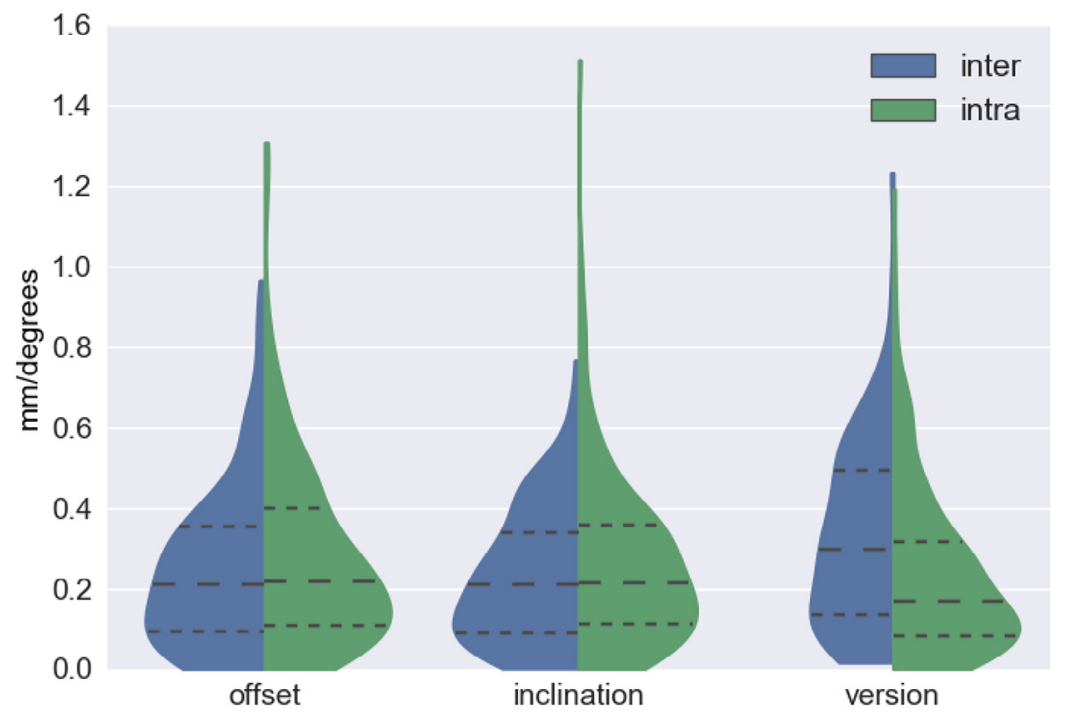

Figure 6 Violin plots showing the distribution of the interoperator (inter) and intraoperator (intra) errors of the 100 scapula. The 0.25 , 0.5 , and 0.75 quartile values are shown by dashed lines.

\section{Conclusion}

This research showed that the contralateral side can guide a surgeon during preoperative planning for the reconstruction of a highly eroded glenoid. Further research should be undertaken to investigate the effect of reconstructing or changing the native anatomy of the scapula in terms of offset, glenoid version, and inclination on shoulder arthroplasty function and survival.

\section{Acknowledgments}

We thank Dr. S. Mufty for help in data processing and measurements.

\section{Disclaimer}

The authors, their immediate families, and any research foundations with which they are affiliated have not received any financial payments or other benefits from any commercial entity related to the subject of this article.

\section{References}

1. Amadi HO, Banerjee S, Hansen UN, Wallace AL, Bull AMJ. An optimised method for quantifying glenoid orientation. Int J Shoulder Surg 2008;2:25-9. http://dx.doi.org/10.4103/0973-6042.41407

2. Bockmann B, Soschynski S, Lechler P, Schwarting T, Debus F, Soca $\mathrm{B}$, et al. The osseous morphology of nondegenerated shoulders shows no side-related differences in elderly patients: an analysis of 102 computed tomography scans. J Shoulder Elbow Surg 2016;25:1297-302. http://dx.doi.org/10.1016/j.jse.2015.12.024

3. Bryce CD, Davison AC, Lewis GS, Wang L, Flemming DJ, Armstrong AD. Two-dimensional glenoid version measurements vary with coronal and sagittal scapular rotation. J Bone Joint Surg Am 2010;92:692-9. http://dx.doi.org/10.2106/JBJS.I.00177

4. Budge MD, Lewis GS, Schaefer E, Coquia S, Flemming DJ, Armstrong AD. Comparison of standard two-dimensional and three-dimensional corrected glenoid version measurements. J Shoulder Elbow Surg 2011;20:577-83. http://dx.doi.org/10.1016/j.jse.2010.11.003

5. Churchill RS, Brems JJ, Kotschi H. Glenoid size, inclination, and version: an anatomic study. J Shoulder Elbow Surg 2001;10:327-32.

6. Crockett HC, Gross LB, Wilk KE, Schwartz ML, Reed J, O’Mara J, et al. Osseous adaptation and range of motion at the glenohumeral joint in professional baseball pitchers. Am J Sports Med 2002;30:20-6. http://dx.doi.org/10.1177/03635465020300011701

7. Daggett M, Werner B, Gauci MO, Chaoui J, Walch G. Comparison of glenoid inclination angle using different clinical imaging modalities. J Shoulder Elbow Surg 2016;25:180-5. http://dx.doi.org/10.1016/ j.jse.2015.07.001

8. Dallalana RJ, McMahon RA, East B, Geraghty L. Accuracy of patientspecific instrumentation in anatomic and reverse total shoulder arthroplasty. Int J Shoulder Surg 2016;10:59-66. http://dx.doi.org/ 10.4103/0973-6042.180717

9. Farron A, Terrier A, Büchler P. Risks of loosening of a prosthetic glenoid implanted in retroversion. J Shoulder Elbow Surg 2006;15:521-6. http://dx.doi.org/10.1016/j.jse.2005.10.003

10. Ganapathi A, McCarron JA, Chen X, Iannotti JP. Predicting normal glenoid version from the pathologic scapula: a comparison of 4 methods in 2- and 3-dimensional models. J Shoulder Elbow Surg 2011;20:234-44. http://dx.doi.org/10.1016/j.jse.2010.05.024

11. Gauci MO, Boileau P, Baba M, Chaoui J, Walch G. Patient-specific glenoid guides provide accuracy and reproducibility in total shoulder arthroplasty. Bone Joint J 2016;98-B:1080-5. http://dx.doi.org/10.1302/ 0301-620X.98B8.37257

12. Ghafurian S, Galdi B, Bastian S, Tan V, Li K. Computerized 3D morphological analysis of glenoid orientation. J Orthop Res 2016;34:692-8. http://dx.doi.org/10.1002/jor.23053

13. Gregory TM, Sankey A, Augereau B, Vandenbussche E, Amis A, Emery $\mathrm{R}$, et al. Accuracy of glenoid component placement in total shoulder 
arthroplasty and its effect on clinical and radiological outcome in a retrospective, longitudinal, monocentric open study. PLoS ONE 2013;8:2-8. http://dx.doi.org/10.1371/journal.pone.0075791

14. Heylen S, Van Haver A, Vuylsteke K, Declercq G, Verborgt O. Patientspecific instrument guidance of glenoid component implantation reduces inclination variability in total and reverse shoulder arthroplasty. J Shoulder Elbow Surg 2016;25:186-92. http://dx.doi.org/10.1016/ j.jse.2015.07.024

15. Ho JC, Sabesan VJ, Iannotti JP. Glenoid component retroversion is associated with osteolysis. J Bone Joint Surg Am 2013;95:e82. http:// dx.doi.org/10.2106/JBJS.L.00336

16. Hoenecke HR, Hermida JC, Flores-Hernandez C, D'Lima DD. Accuracy of CT-based measurements of glenoid version for total shoulder arthroplasty. J Shoulder Elbow Surg 2010;19:166-71. http://dx.doi.org/ 10.1016/j.jse.2009.08.009

17. Jacobson SR, Mallon WJ. The glenohumeral offset ratio: a radiographic study. J Shoulder Elbow Surg 1993;2:141-6.

18. Jeske H-C, Oberthaler M, Klingensmith M, Dallapozza C, Smekal V, Wambacher M, et al. Normal glenoid rim anatomy and the reliability of shoulder instability measurements based on intrasite correlation. Surg Radiol Anat 2009;31:623-5. http://dx.doi.org/10.1007/s00276-009-0492-0

19. Karelse A, Van Tongel A, Verstraeten T, Poncet D, De Wilde LF. Rocking-horse phenomenon of the glenoid component: the importance of inclination. J Shoulder Elbow Surg 2015;24:1142-8. http://dx.doi.org/ 10.1016/j.jse.2014.12.017

20. Kwon YW, Powell KA, Yum JK, Brems JJ, Iannotti JP. Use of threedimensional computed tomography for the analysis of the glenoid anatomy. J Shoulder Elbow Surg 2005;14:85-90. http://dx.doi.org/ 10.1016/j.jse.2004.04.011

21. Matsumura N, Ogawa K, Kobayashi S, Oki S, Watanabe A, Ikegami $\mathrm{H}$, et al. Morphologic features of humeral head and glenoid version in the normal glenohumeral joint. J Shoulder Elbow Surg 2014;23:1724-30. http://dx.doi.org/10.1016/j.jse.2014.02.020

22. Maurer A, Fucentese SF, Pfirrmann CWA, Wirth SH, Djahangiri A, Jost $\mathrm{B}$, et al. Assessment of glenoid inclination on routine clinical radiographs and computed tomography examinations of the shoulder. J Shoulder Elbow Surg 2012;21:1096-103. http://dx.doi.org/10.1016/j.jse.2011 .07 .010

23. Nyffeler RW, Jost B, Pfirrmann CWA, Gerber C. Measurement of glenoid version: conventional radiographs versus computed tomography scans. J Shoulder Elbow Surg 2003;12:493-6. https://doi.org/10.1016/S1058 $-2746(03) 00181-2$

24. Oosterom R, Rozing PM, Bersee HEN. Effect of glenoid component inclination on its fixation and humeral head subluxation in total shoulder arthroplasty. Clin Biomech (Bristol, Avon) 2004;19:1000-8. http:// dx.doi.org/10.1016/j.clinbiomech.2004.07.001
25. Sirveaux F, Favard L, Oudet D, Huquet D, Walch G, Molé D. Grammont inverted total shoulder arthroplasty in the treatment of glenohumeral osteoarthritis with massive rupture of the cuff. Results of a multicentre study of 80 shoulders. J Bone Joint Surg Br 2004;86:388-95. http:// dx.doi.org/10.1302/0301-620X.86B3.14024

26. Throckmorton TW, Gulotta LV, Bonnarens FO, Wright SA, Hartzell JL, Rozzi WB, et al. Patient-specific targeting guides compared with traditional instrumentation for glenoid component placement in shoulder arthroplasty: a multi-surgeon study in 70 arthritic cadaver specimens. J Shoulder Elbow Surg 2015;24:965-71. http://dx.doi.org/10.1016/ j.jse.2014.10.013

27. van de Bunt F, Pearl ML, Lee EK, Peng L, Didomenico P. Glenoid version by CT scan: an analysis of clinical measurement error and introduction of a protocol to reduce variability. Skeletal Radiol 2015;44:1627-35. http://dx.doi.org/10.1007/s00256-015-2207 $-4$

28. Van den Broeck J, Vereecke E, Wirix-Speetjens R, Vander Sloten J. Segmentation accuracy of long bones. Med Eng Phys 2014;36:949-53. http://dx.doi.org/10.1016/j.medengphy.2014.03.016

29. Van Haver A, Heylen S, Vuylsteke K, Declercq G, Verborgt O. Reliability analysis of glenoid component inclination measurements on postoperative radiographs and computed tomography-based 3D models in total and reversed shoulder arthroplasty patients. J Shoulder Elbow Surg 2016;25:632-40. http://dx.doi.org/10.1016/j.jse.2015.09.003

30. Victor J, Van Doninck D, Labey L, Innocenti B, Parizel PM, Bellemans J. How precise can bony landmarks be determined on a CT scan of the knee? Knee 2009;16:358-65. http://dx.doi.org/10.1016/j.knee.2009.01.001

31. von Schroeder HP, Kuiper SD, Botte MJ. Osseous anatomy of the scapula. Clin Orthop Relat Res 2001;383:131-9.

32. Walch G, Badet R, Boulahia A, Khoury A. Morphologic study of the glenoid in primary glenohumeral osteoarthritis. J Arthroplasty 1999;14:756-60.

33. Walch G, Young AA, Melis B, Gazielly D, Loew M, Boileau P. Results of a convex-back cemented keeled glenoid component in primary osteoarthritis: multicenter study with a follow-up greater than 5 years. J Shoulder Elbow Surg 2011;20:385-94. http://dx.doi.org/10.1016/ j.jse.2010.07.011

34. Welsch G, Mamisch TC, Kikinis R, Schmidt R, Forst R, Fitz W, et al. CT-based preoperative analysis of scapula morphology and glenohumeral joint geometry. Comput Aided Surg 2003;8:264-8. http://dx.doi.org/ $10.3109 / 10929080309146062$

35. Wu G, van der Helm FCT, Veeger HEJD, Makhsous M, Van Roy P, Anglin C, et al. ISB recommendation on definitions of joint coordinate systems of various joints for the reporting of human joint motion-part II: shoulder, elbow, wrist and hand. J Biomech 2005;38:981-92. http://dx.doi.org/10.1016/j.jbiomech.2004.05.042 